in the $\alpha / R^{3}$ term as Gunzig et al. suppose. Their calculation could be used to estimate the lepto-quark density of grand unified theories, but certainly not the density of any of the present constituents of matter.

We stress that the field-theoretical foundations of the production mechanism as well as the instability of Minkowski space are flimsy, at best - though not necessarily wrong - owing to the use of an unconventional and arbitrary subtraction procedure ${ }^{2}$. The model of inflation through black hole production ${ }^{2}$ must be regarded as a hypothesis without solid theoretical support. If such exists, it must await the yet-to-come quantum theory of gravity. To our mind, any numerical evaluation, unless it definitely detroys the model, is premature. (A more detailed account of our calculations is available in ref. 3 or from us).

R. BRout PH. SPINDEL.

Service de Physique Theorique,

Université Libre de Bruxelles,

Plaine Campus (C.P. 225),

B-1050 Bruxelles, Belgium

GunZIG REPLIES-Brout and Spindel claim that our paper ${ }^{1}$ contains several "errors". We firmly disagree with this conclusion. I will consider their criticisms.

The first point concerns the matching parameters $\alpha$ and $\beta$. We agree entirely with their remark concerning the sensitivity of $\alpha$ to the input. But they miss the key point, which is that there is a subtle compensation of infinitesimals which leads to our result $\alpha / \sqrt{\beta}=0(1)$ : it appears indeed that the solution of the matching equations (14) and (15) is given by $\alpha / \sqrt{\beta}=1-\varepsilon / \varepsilon_{1}$, where $\varepsilon=$ $\left(\beta / C^{2}\right)-1$ and $\varepsilon_{1}=1-\Delta_{1} \approx 10^{-27}$. It results straightforwardly from the definitions of the parameters $\beta$ and $C$, that $\beta / C^{2}=1 / \Delta_{1}\left(T / T_{\mathrm{BH}}\right)^{4}$. The value of $T / T_{\mathrm{BH}}$ is easily evaluated in the euclidean parameterization of the joining conditions; the latter is appropriate as the joining point belongs to the de Sitter asymptotic region. It appears that $\left(T / T_{\mathrm{BH}}\right)^{4}$ $=1-\varepsilon_{2}$ with $\varepsilon_{2} / \varepsilon_{1}=0(1)$. Hence,

$$
\alpha /, \bar{\beta}=\left[1-\frac{\left(\varepsilon_{1}-\varepsilon_{2}\right)}{\varepsilon_{1}}\right]=\frac{\varepsilon_{2}}{\varepsilon_{1}}=0(1)
$$

as we announced'. Moreover, when the cosmogenesis model is wholly reformulated in the framework of euclidean Robertson-Walker parameterization, as we did recently ${ }^{4}$, the matching conditions give rise to much simpler algebraic relations than those associated with the open Robertson-Walker parameterization. These relations lead straightforwardly to conclusions identical to those resulting from the $\simeq \sqrt{\beta}$ open condition.

The second point concerns the number of helicity states involved in our considerations. We mentioned this problem at the time'. Since then, we have shown ${ }^{4}$

that the inclusion of any higher number of helicity states leaves all the results unaltered, provided that the black hole mass $M$ is increased (by a factor of about 5 for $N=100$, for example).

The third criticism concerns the use of the proton mass in the evaluation of the specific entropy. First of all, as mentioned in our paper ${ }^{1}$, the fundamental quantity to be considered is the adiabatic invariant $(1 / T)\left(\varrho_{y} / \varrho_{m}\right)$ which does not involve any elementary particle mass at all. Our calculations produce the correct experimental value for this quantity as well. Nevertheless, the quantity which is traditionally discussed in the literature is the specific entropy per proton. To obtain the latter, it is fully legitimate in our context to use the proton mass. Indeed our joining procedure connects directly the de Sitter stage to the present cosmological stage. We mentioned in our conclusions that in a more realistic model, our parameters $\alpha$ and $\beta$ would be time-dependent.

In addition to these three specific criticisms, Brout and Spindel are pessimistic about the field-theoretical foundations of the production mechanism as well as the

\title{
Selective action of artificial membranes
}

SIR-Rubinstein et al. ${ }^{1}$ claim to have prepared the first example of a stable, ionselective artificial membrane of monolayer thickness, which successfully mimics basic structural and functional properties of natural bilayer membranes. Their membrane was prepared on a gold electrode by coadsorption of two components from bicyclohexyl/chloroform solution. After transfer to an aqueous solution, the first of these (OM) apparently acted as a blocking agent, and the second (TBEA) as an 'active' ligand forming 1:1 complexes with divalent metal ions, such as $\mathrm{Cu}^{2+}$, but not with travalent ions, such as $\mathrm{Fe}^{3+}$.

To establish that their 'artificial membrane' was working as they claimed, the authors needed to prove that the only observed electrochemical reaction was caused by the active ligand chemically distinguishing between $\mathrm{Cu}^{2+}$ and $\mathrm{Fe}^{3+}$ ions in solution. Although certain evidence was adduced in favour of this hypothesis, we believe that there is an alternative and much simpler interpretation of the experimental results in which the active ligand has no role.

When the 'artificial membrane' was placed in a solution of $\mathrm{Cu}^{2+}$ and $\mathrm{Fe}^{3+}$ ions, Rubinstein et al. observed no $\mathrm{Cu}^{2+}$ 'underpotential' (non-bulk) and $\mathrm{Fe}^{2+} / \mathrm{Fe}^{3+}$ peaks; repression of the peak for bulk deposition of copper onto the electrode by $\sim 250 \mathrm{mV}$; and a loop in the cyclic voltammetry. Rubinstein et al. interpret these observations as follows: $\mathrm{Cu}^{2+}$ peaks were absent because copper atoms were deposited inside the 'artificial membrane' at some distance from, rather than directly on, the instability of Minkowski space. These remarks are directed towards the series of papers on the subject, initiated by Brout, Englert and myself ${ }^{s}$. I share this concern. It is for this very reason that we have now developed $\mathrm{d}^{4}$ a phenomenological approach which eliminates the field theoretical subtleties. In this context, simple thermodynamical arguments render the explicit use of field-theoretical considerations no longer necessary.

In conclusion, Brout and Spindel have missed the main points of our paper. These involve the reinterpretation of Einstein's equations leading to a burst of entropy associated with matter creation in the early stages of the Universe.

Service de Chimie Physique II, E. GunzIG

Université Libre de Bruxelles,

Campus Plaine (C.P. 231),

B-1050 Bruxelles, Belgium

1. E. Gunzig, et al. Nature 330, 621- 624 (1987).

2. F. Englert in Les Houches Session XXXII 516 (NorthHolland, Amsterdam, 1980).

3. Brussels Report ULB-TH 88/05.

4. Prigogine, I. et al. Proc. natn. Acad. Sci. U.S.A. 85

7428-7432 (1988).

5. Brout, R. et al. E. Ann. Phys. 113, 78-106 (1978). electrode surface; the lack of $\mathrm{Fe}^{2+} / \mathrm{Fe}^{3+}$ peaks was due to a lack of sites where $\mathrm{Fe}^{3+}$ ions could bind; and the shift in the bulk copper peak and the existence of the voltammetric loop were evidence of a "preferred perpendicular orientation of the ligand". Furthermore, they invoke quantum-mechanical tunnelling barrier to explain the shift of the copper deposition peak, and suggest a type of autocatalysis to explain the voltammetric loop.

But all the above evidence is also consistent with a simple physical model in which $\mathrm{Cu}^{2+}$ ions are discharged directly onto the surface of the gold electrode. The electrode may be imagined as being, say, only $99.9 \%$ covered by the monolayer and not $100 \%$ as claimed. Such a coverage would be sufficient to eliminate all but $0.1 \%$ of both the copper 'underpotential' and $\mathrm{Fe}^{2+} / \mathrm{Fe}^{3+}$ peaks, making them undetectable on the scale used, but would leave enough area of uncovered gold to allow the nucleation and growth of bulk copper crystals. Moreover, the high coverage would also block a large fraction of the active sites for nucleation on the gold surface, so that the nucleation overpotential for copper deposition would be increased by several hundred millivolts, precisely as observed. The loops in the voltammograms are no more than the expected response of a nucleation-andgrowth process to a triangular scan of applied potential, as proved elsewhere ${ }^{2}$.

STEPHEN FleTCHER CSIRO Division of Mineral Products,

PO Box 124, Port Melbourne,

Victoria 3207, Australia 